\title{
Benchmarks
}

\section{Precise breakpoint localization of large genomic deletions using PacBio and Illumina next-generation sequencers}

\author{
Michal J Okoniewski ${ }^{1,2 *}$, Janine Meienberg ${ }^{3,4 *}$, Andrea Patrignani ${ }^{1}$, Alicja Szabelska ${ }^{1,5}$, Gabor Matyas ${ }^{3,4, \#}$, Ralph \\ Schlapbach ${ }^{1, \#}$ \\ ${ }^{1}$ Functional Genomics Center Zurich, Zurich, Switzerland, ${ }^{2}$ Department of Neuroimmunology and Multiple \\ Sclerosis Research, Neurology Clinic, University Hospital, Zurich, Switzerland, ${ }^{3}$ Center for Cardiovascular Genetics \\ and Gene Diagnostics, Zurich, Switzerland, ${ }^{4}$ Zurich Center of Integrative Human Physiology, University of Zurich, \\ Zurich, Switzerland, ${ }^{5}$ Department of Mathematical and Statistical Methods, Poznan University of Life Sciences, \\ Poznan, Poland
}

*These authors contributed equally to this work

\#These authors jointly directed this work

Keywords: sequencing, large deletions, amplicons, PacBio, Illumina, Sanger

Supplementary material for this article is available at www.BioTechniques.com/article/113992.

Herein we present the applicability of single-molecule (PacBio RS) and second-generation sequencing technology (Illumina) to the characterization of large genomic deletions. By testing samples previously characterized using a Sanger approach, our methods determined that both next-generation sequencing platforms were able to identify the position of deletion breakpoints. Our results point out various advantages of next-generation sequencing platforms when characterizing genomic deletions; however, special attention must be dedicated to identical sequences flanking the breakpoints, such as poly $(\mathrm{N})$ motifs.

The PacBio RS next-generation sequencing (NGS) technology (Pacific Biosciences, Menlo Park, CA, USA) has not only the potential to identify modified bases and thereby characterize methylation patterns $(1,2)$, but it also provides previously unprecedented sequencing read lengths $(>2 \mathrm{~kb})$, making it useful for quickly improving existing genome assemblies (3). In this study, we used the advantage of such long reads for the characterization of large deletions previously identified by multiplex ligationdependent probe amplification (MLPA) and microarray analyses. Using traditional Sanger sequencing to characterize large deletions is time-consuming and work-intensive $(4,5)$, increasing the need for effective breakpoint localization. Indeed, for Sanger sequencing a large fragment $(2-10 \mathrm{~kb})$ containing the breakpoints has to be amplified by longrange PCR (LR-PCR) and subsequently sequenced in order to identify exact breakpoint positions. Furthermore, since Sanger sequencing permits only $\sim 600$ bp to be sequenced using one primer, several sets of internal primers are required for large LR-PCR products.

In contrast, NGS may offer simplified sequencing in such cases. Herein, we tested this possibility by using not only the long reads of the PacBio platform, but also the short reads of a second-generation sequencing technology (HiSeq 2000, Illumina, San Diego, CA, USA). Illumina offers stable lengths of short reads (100 bp in this case) with errors most likely to be grouped at the ends of reads $(6,7)$; the PacBio RS reads from this study had a mean length of $2459 \mathrm{bp}$ and random distribution of errors affecting 10-15\% of nucleotides. In addition, only a few dedicated computational techniques are available for the characterization of large deletions by NGS (8), making data analysis a challenge.

The three DNA samples used in this study harbor previously characterized large hemizygous deletions. Deletions in sample 44 and sample 70 (of length 26,887 bp and 302,580 bp, respectively) affect the FBN1 gene in patients with Marfan syndrome (4); a deletion in sample 53B has a size of $3,408,306$ bp and comprises the entire COL $3 A 1$ gene in a patient with EhlersDanlos syndrome vascular type (5). Accordingly, 6.5-8.5 kb LR-PCR products were amplified using the Expand Long Template PCR System (Roche Diagnostics, Rotkreuz, Switzerland) as described previously $(4,5)$ and purified by means of QIAquick PCR Purification Kit (Qiagen, Hilden, Germany).

SMRTbell libraries were prepared using the PacBio C2 chemistry (3-10 kb) DNA preparation kit (Part no. 001-540-726,

\section{Method summary:}

We present the applicability of single-molecule (PacBio RS) and second-generation sequencing technology (Illumina) to the characterization of large genomic deletions. Both next-generation sequencing platforms were able to identify the position of deletion breakpoints previously identified by Sanger sequencing. 


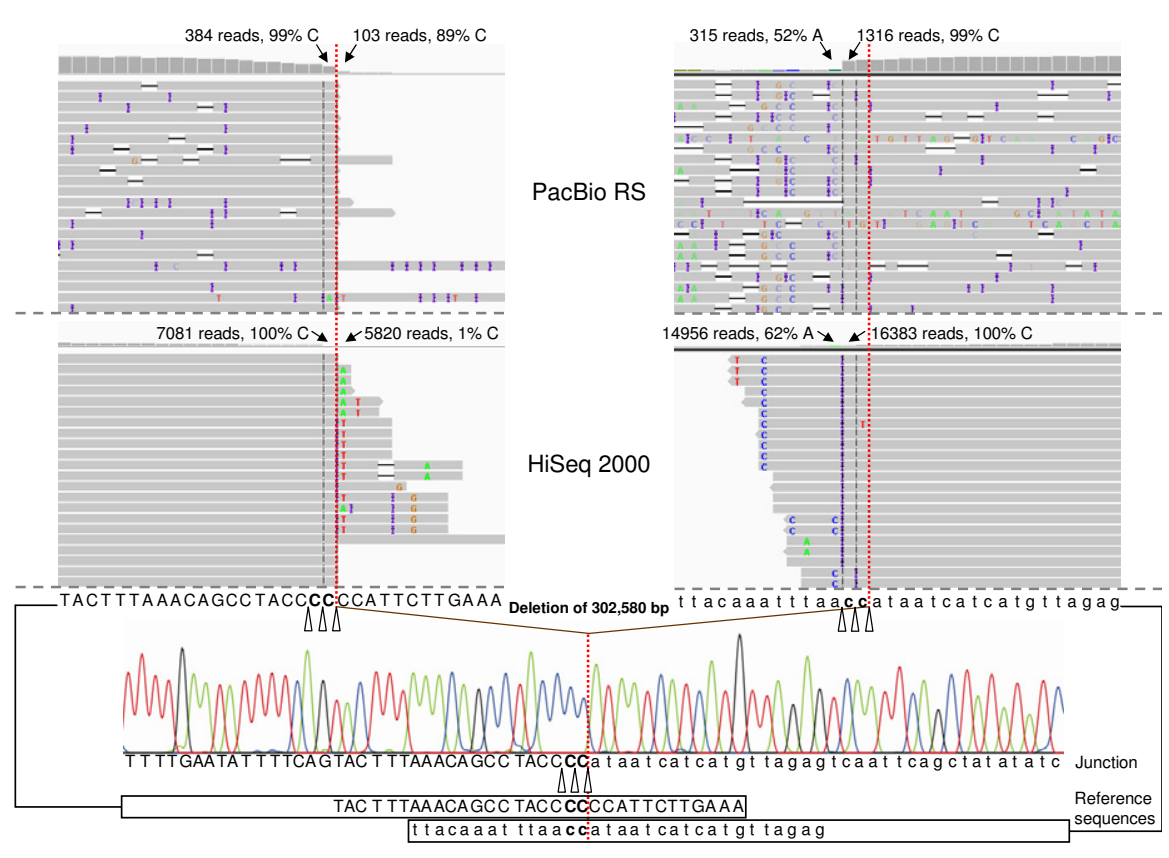

Figure 1. Both ends of the 302,580-bp deletion on chromosome 15 in sample 70 displayed in the Integrative Genomics Viewer. Aligned reads are displayed as gray bars/arrows; letters indicate mismatched bases; purple vertical dashes indicate insertions; and black horizontal lines indicate deletions. The reads generated by PacBio RS (upper panel) and Illumina HiSeq 2000 (lower panel) were sorted by aligned position, base, and mapping quality, and compared with the results of Sanger sequencing (bottom). Sections of 22 reads are shown. The top tracks show a much clearer decrease in the PacBio reads, whereas the lllumina reads show a clearer increase in mismatches at the sites of breakpoints. Uppercase letters represent the sequence in the region of the start point of the deletion; lowercase letters represent the sequence in the region of the deletion end point. Due to identical sequences at the sites of breakpoints, the break and re-joining could have occurred at three positions, as indicated by open triangles. The dotted red line marks the most telomeric position of the possible breakpoints. Note that the total read counts (reads) and the percentage of reference bases (\%) are given for the positions flanking the site where the coverage (gray bars) starts to lower. For more details, see Tables 1 and 2 .

Pacific Biosciences) as well as $5 \mu \mathrm{g}$ purified amplicons without fragmentation. Libraries were subsequently sequenced on the PacBio RS using one SMRT cell per sample and taking two movies of 45 min each. The reads have been mapped with the BLASR mapper (9), which is supplied in the SMRT Portal software suite (Pacific Biosciences) and applies therefore as a standard mapper for PacBio reads. The same amplicons were sequenced on the HiSeq 2000 sequencer using Illumina's TruSeq DNA Sample Preparation v2 protocol with $1 \mu \mathrm{g}$ input material and $100+100 \mathrm{bp}$ pair-end reads. The reads were mapped using the standard mapper, Bowtie (10). For both NGS platforms, the mappers have been used with default parameters. Respective sequences are available in the NCBI Sequence Read Archive (object ID: ERP002092).

For the PacBio data, the read coverage in the SMRT Portal software suite resulted in a clear drop of read depth in the deleted region (see Supplementary Figure S5), which was subsequently confirmed by zooming in on the breakpoint regions by means of the Integrative Genomics Viewer (IGV) (11) (Figure 1 and Tables 1 and 2 for sample 70; see Supplementary Figures S1-S2 as well as Supplementary Tables S1-S4 for samples 44 and 53B, respectively). Respective Illumina data displayed in IGV showed more gradually sinking patterns at the expected deletion ends; the site of breakpoints in these data was identified by an increase in mismatches (Figure 1, Tables 1 and 2, Supplementary Figures S1-S2, and Supplementary Tables S1-S4). This may be due to the fact that the mappers typically allow several mismatches, meaning that many of the short Illumina reads could be mapped over the breakpoints. In contrast, PacBio RS data show a number of reads spanning over the deletion, which have not been mapped by the SMRT Portal aligner to the standard reference due to the high number of mismatches. The read depth of both platforms is more than sufficient to find the breakpoint; tests using $1 / 2$ or $1 / 3$ of reads per sample also produced satisfactory results (data not shown).

An additional difficulty may be identical sequences on both sides of the deletion, a common phenomenon that has already been described for different genes (12-14).

Table 1. Read depth and percentage of the wild-type allele in the region flanking the breakpoint at the start site of the deletion in sample 70.

\begin{tabular}{|cccccccccccc|}
\hline Location & \multicolumn{9}{c}{ Not deleted } & \multicolumn{1}{c|}{ Deleted } \\
\hline $\begin{array}{l}\text { Wild-type } \\
\text { Sequence }\end{array}$ & A & C & C & C & C & C & C & A & T & T \\
\hline PacBio RS & $614(100 \%)$ & $531(100 \%)$ & $508(100 \%)$ & $492(100 \%)$ & $384(99 \%)$ & $103(89 \%)$ & $66(88 \%)$ & $66(98 \%)$ & $61(95 \%)$ & $29 *(86 \%)$ \\
& 12699 & 11679 & 9545 & 8278 & 7081 & $\vdots$ & 5820 & 4313 & 3224 & 1678 & $1536 * *$ \\
HiSeq 2000 & $(100 \%)$ & $(100 \%)$ & $(100 \%)$ & $(100 \%)$ & $(100 \%)$ & $\vdots$ & $(1 \%)$ & $(48 \%)$ & $(100 \%)$ & $(99 \%)$ & $(98 \%)$ \\
\hline
\end{tabular}

*No mapped reads 243 bases after the most telomeric breakpoint (read depth $=0$ ). ** No mapped reads 117 bases after the most telomeric breakpoint (read depth $=0$ ). Bold letters indicate identical bases at the sites of the breakpoint, which can be either up- or downstream of the breakpoint. The red dotted line indicates the most telomeric position of the three possible breakpoints. This is also the point where the read depth drops and the number of mismatches increases (see Figure 1 ).

Table 2. Read depth and percentage of the wild-type allele in the region flanking the breakpoint at the end of the deletion in sample 70.

\begin{tabular}{|c|c|c|c|c|c|c|c|c|c|c|}
\hline Location & & & Deleted & & & & & Not deleted & & \\
\hline $\begin{array}{l}\text { Wild-type } \\
\text { Sequence }\end{array}$ & $\mathrm{t}$ & $\mathrm{t}$ & $t$ & a & a & c & c & a & $\mathrm{t}$ & a \\
\hline PacBio RS & $231 *(8 \%)$ & 242 (10\%) & 251 (94\%) & 215 (95\%) & 315 (52\%) & 1316 (99\%) & 1359 (99\%) & $1411(99 \%)$ & 1465 (99\%) & 1547 (100\%) \\
\hline $\begin{array}{l}\text { HiSeq } \\
2000\end{array}$ & $\begin{array}{l}5408 * * \\
(100 \%)\end{array}$ & $\begin{array}{l}8762 \\
(87 \%)\end{array}$ & $\begin{array}{l}10427 \\
(83 \%)\end{array}$ & $\begin{array}{l}13679 \\
(92 \%)\end{array}$ & $\begin{array}{l}14956 \\
(62 \%)\end{array}$ & $\begin{array}{c}16383 \\
(100 \%)\end{array}$ & $\begin{array}{l}17735 \\
(96 \%)\end{array}$ & $\begin{array}{c}18658 \\
(100 \%)\end{array}$ & $\begin{array}{c}19512 \\
(100 \%)\end{array}$ & $\begin{array}{c}20549 \\
(100 \%)\end{array}$ \\
\hline
\end{tabular}

* No mapped reads 210 bases before the most telomeric breakpoint (read depth $=0$ ). ${ }^{*}$ No mapped reads 16 bases before the most telomeric breakpoint $($ read depth $=0$ ). Bold letters indicate identical bases at the sites of breakpoints, which can be either up- or downstream of the breakpoint. The red dotted line indicates the most telomeric position of the possible breakpoints. The most centromeric breakpoint, where the read depth drops and the number of mismatches increases, is indicated by a black bold line (see Figure 1). 


\begin{tabular}{|c|c|c|c|c|c|c|c|c|c|c|}
\hline \multicolumn{11}{|l|}{ PacBio } \\
\hline & $\mathrm{n}=0$ & $n=1$ & $n=2$ & $n=3$ & $n=4$ & $n=5$ & $n=6$ & $n=7$ & $n=8$ & type II error \\
\hline Flank 5 & 56 & 28 & 191 & 941 & 2132 & 348 & 37 & 8 & 0 & $3 e-141$ \\
\hline Flank 10 & 0 & 1 & 53 & 329 & 820 & 124 & 12 & 3 & 0 & $1 e-46$ \\
\hline Flank 20 & 0 & 0 & 5 & 56 & 127 & 19 & 2 & 1 & 0 & $5 e-06$ \\
\hline \multicolumn{11}{|l|}{ Illumina } \\
\hline & $n=0$ & $n=1$ & $n=2$ & $n=3$ & $n=4$ & $n=5$ & $n=6$ & $n=7$ & $n=8$ & type II error \\
\hline Flank 5 & 2 & 3 & 0 & 29 & 68817 & 3 & 0 & 0 & 0 & $<2 e-308$ \\
\hline Flank 10 & 0 & 0 & 0 & 20 & 58769 & 0 & 0 & 0 & 0 & $<2 e-308$ \\
\hline Flank 20 & 0 & 0 & 0 & 13 & 43258 & 0 & 0 & 0 & 0 & $<2 e-308$ \\
\hline
\end{tabular}

\section{ICAGTACIIIAAACAGCCTA(C)nATAATCATCATGIIAGAGTC.}

Figure 2. Counts of exact matches for different lengths of a poly(C) motif (red) at the site of deletion breakpoints in sample $\mathbf{7 0}$ with 5-, 10-, and 20-nucleotide flanking sequences for both PacBio and Illumina reads. The count indicates that the true sequence includes $4 \times C(n=4)$ (see Figure 1). Corresponding type II errors were calculated using the R script provided in the Supplementary Materials.

In particular, this could be observed in all three deletions presented in this study ("CC" in samples 53B and 70 and "GC" in sample 44). In order to find the precise sequence of poly $(\mathrm{N})$ motifs (tandemly repeated nucleotides) at the sites of break and rejoining, we have developed an AWK script to count matches at the sites of suspected deletion breakpoints (see Supplementary Materials). This counting was performed with perfect matches only, resulting in the data depicted in Figure 2 (sample 70) and Supplementary Figures S3 and S4 (samples 44 and 53B, respectively). When a single nucleotide (or pair, in the case of GC) has a fixed probability of being misinterpreted, it can be assumed without loss of generality that the distribution of the occurrences of specific motifs follows the Poisson distribution. The hypothesis that the maximum number of counts represents the appropriate motif has been tested. For PacBio RS reads in sample 70 , the probabilities of wrongly accepting the null hypothesis are far below the 0.01 level of significance $\left(p=1.5 \times 10^{-23}, p=1.06 \times 10^{-46}\right.$, and $p=3.2 \times 10^{-141}$ in the cases of 20,10 , and 5 flanking bases, respectively) (Figure 2). In the case of Illumina, due to the high number of reads, error levels are so low that they go below that afforded by the smallnumber precision in the $\mathrm{R}$ language. For details on the calculations, see the R script in the Supplementary Materials. The script can be used on any FASTA or FASTQ data and checks the statistical power at a given significance level regardless of the platform.

As shown by this study, the determination of deletion breakpoints can be done with data obtained from both NGS platforms. However, whereas the long reads of PacBio RS showed a sharp decrease in read depth, Illumina short reads exhibited an increase in mismatches related to the position of the breakpoints. Sample preparation costs are comparable for PacBio and Illumina platforms. However, sequencing using PacBio RS can be done within a working day, while Illumina's system, even the smaller MiSeq version, requires more time. Both platforms are suitable for precise breakpoint localization, provide an alternative procedure for the characterization of large deletions, and require fewer resources and less time than traditional Sanger sequencing.

\section{Acknowledgments}

We are grateful to Yu-Chih Tsai, Jonas Korlach, and Stephen W. Turner for discussions on PacBio technology and data analysis. This work was supported by the FGCZ, as well as grants from the COFRA Foundation (to G.M.), Gottfried \& Julia BangerterRhyner-Stiftung (to G.M.), Jubiläumsstiftung Swiss Life (to G.M.), Foundation for People with Rare Diseases (to J.M. and G.M.), Clinical Research Priority Program (CRPP/ KFSP-MS) of University of Zurich (to M.O.), and Sciex.ch (no. 11.182 to A.S. and M.O.).

\section{Competing interests}

The authors declare no competing interests.

\section{References}

1. Clark, T.A., I.A. Murray, R.D. Morgan, A.O. Kislyuk, K.E. Spittle, M. Boitano, A. Fomenkov, R.J. Roberts, and J. Korlach. 2012. Characterization of DNA methyltransferase specificities using single-molecule, real-time

2. Murray, I.A., T.A. Clark, R.D. Morgan, M. Boitano, B.P. Anton, K. Luong, A. Fomenkov, S.W. Turner, et al. 2012. The methylomes of six bacteria. Nucleic Acids Res. 40:11450-11462. DNA sequencing. Nucleic Acids Res. 40:e29.
3. Zhang, X., K.W. Davenport, W. Gu, H.E. Daligault, A.C. Munk, H. Tashima, K. Reitenga, L.D. Green, and C.S. Han. 2012. Improving genome assemblies by sequencing PCR products with PacBio. BioTechniques 53:61-62.

4. Mátyás, G., S. Alonso, A. Patrignani, M. Marti, E. Arnold, I. Magyar, C. Henggeler, T. Carrel, et al. 2007. Large genomic fibrillin-1 (FBN1) gene deletions provide evidence for true haploinsufficiency in Marfan syndrome. Hum. Genet. 122:23-32.

5. Meienberg, J., M. Rohrbach, S. Neuenschwander, K. Spanaus, C. Giunta, S. Alonso, E. Arnold, C. Henggeler, et al. 2010. Hemizygous deletion of COL3A1, COL5A2, and MSTN causes a complex phenotype with aortic dissection: a lesson for and from true haploinsufficiency. Eur. J. Hum. Genet. 18:1315-1321.

6. Kozarewa, I., Z. Ning, M.A. Quail, M.J. Sanders, M. Berriman, and D.J. Turner. 2009. Amplification-free Illumina sequencing-library preparation facilitates improved mapping and assembly of (G+C)-biased genomes. Nat. Methods 6:291-295.

7. McElroy, K.E., F. Luciani, and T. Thomas. 2012. GemSIM: general, error-model based simulator of next-generation sequencing data. BMC Genomics 13:74.

8. Ye, K., M.H. Schulz, Q. Long, R. Apweiler, and $Z$. Ning. 2009. Pindel: a pattern growth approach to detect break points of large deletions and medium sized insertions from paired-end short reads. Bioinformatics 25:2865-2871.

9. Chaisson, M.J. and G. Tesler. 2012. Mapping single molecule sequencing reads using Basic Local Alignment with Successive Refinement (BLASR): Theory and application. BMC Bioinformatics 13:238.

10. Langmead, B. 2010. Aligning short sequencing reads with Bowtie. Curr. Protoc. Bioinformatics Chapter 11:Unit 11.7.

11. Thorvaldsdottir, H., J.T. Robinson, and J.P. Mesirov. 2012. Integrative Genomics Viewer (IGV): high-performance genomics data visualization and exploration. Brief Bioinform [Epub ahead of print].

12. Giacalone, J.P. and U. Francke. 1992. Common sequence motifsat the rearrangementsites of a constitutional X/autosome translocation and associated deletion. Am.J. Hum. Genet. 50:725-741.

13. Otto, E., R. Betz, C. Rensing, S. Schatzle, T. Kuntzen, T. Vetsi, A. Imm, and F. Hildebrandt. 2000. A deletion distinct from the classical homologous recombination of juvenile nephronophthisis type 1 (NPH1) allows exact molecular definition of deletion breakpoints. Hum. Mutat. 16:211-223.

14. Liu, H.X., L. Cartegni, M.Q. Zhang, and A.R. Krainer. 2001. A mechanism for exon skipping caused by nonsense or missense mutations in BRCA1 and other genes. Nat. Genet. 27:55-58.

Received 26 October 2012; accepted 8 January 2013.

Address correspondence to Michal J. Okoniewski, FGCZ, Winterthurerstrasse 190, 8057 Zurich, Switzerland. Email: michal.okoniewski@fgcz.ethz.ch

To purchase reprints of this article, contact: biotechniques@fosterprinting.com 